\title{
CUIDADORES FAMILIARES DOS SOBREVIVENTES DE ACIDENTE VASCULAR CEREBRAL: SOBRECARGA E FATORES RELACIONADOS
}

\author{
FAMILY CAREGIVERS OF STROKE SURVIVORS: BURDEN AND \\ RELATED FACTORS
}

\begin{abstract}
CUIDADORES FAMILIARES DE SOBREVIVIENTES DE ACCIDENTE CEREBROVASCULAR: SOBRECARGA Y FACTORES RELACIONADOS
\end{abstract}

\author{
Jaine Kareny Da Silva* \\ Rita Narriman Silva De Oliveira Boery**
}

\begin{abstract}
RESUMO
Objetivo: Analisar a sobrecarga dos cuidadores familiares dos sobreviventes de Acidente Vascular Cerebral (AVC) e os fatores relacionados. Material e Método: Estudo transversal e correlacional, realizado com uma amostra não probabilística, selecionada por conveniência, de cuidadores familiares dos sobreviventes de AVC, atendidos em 17 Unidades Básicas de Saúde da zona urbana ou fossem atendidos na única unidade hospitalar pública do município do interior da Bahia entre janeiro de 2014 à agosto de 2017. Os dados foram coletados entre setembro de 2017 e março de 2018 nas residências dos cuidadores, a partir de um formulário sociodemográfico e das escalas de Katz, de Zarit Burden Interview e do Perceived Stress, que foram analisados pela estatística descritiva e pelo coeficiente de correlaçáo de Spearman. Resultados: Os 44 cuidadores familiares apresentam sobrecarga de leve a moderada, que se relacionou significativamente com o estresse do cuidador e o grau de dependência do sobrevivente de AVC. Conclusão: Este dado evidencia a necessidade de maior apoio social formal aos sobreviventes de AVC e seus cuidadores familiares, por meio de açóes em saúde que favorecem a independência daquelas pessoas com sequelas do AVC e incluam o cuidador no plano de reabilitaçáo, sobretudo no âmbito emocional, com o intuito de reduzir a sobrecarga do cuidador, melhorando, assim, o bem-estar desses indivíduos.
\end{abstract}

Palavras-chave: Acidente vascular cerebral; Cuidadores; Família; Cuidados de Enfermagem; Carga de trabalho.

Financiamento: Bolsa do Programa de Apoio à Capacitação de Docentes e Técnicos Administrativos da UNEB (PAC-DT).

*Doutora. Universidade do Estado da Bahia, Campus XII, Guanambi, Bahia, Brasil. ORCID: http://orcid.org/0000-0002-84878384 Email: jainekareny@yahoo.com.br Autora correspondente.

** Doutora. Programa de pós-graduação em Enfermagem e Saúde, Universidade Estadual do Sudoeste Bahia, Campus Jequié, Jequié, Bahia, Brasil. ORCID: http://orcid.org/0000-0002-7823-9498 Email: rboery@uesb.edu.br 


\section{ABSTRACT}

Objective: To analyze the burden of family caregivers of stroke survivors and related factors. Materials and Methods: Cross-sectional and correlational study, carried out with a non-probabilistic sample, selected by convenience, of family caregivers of stroke survivors, who were assisted in 17 primary health care units of the urban area or in the only public hospital unit of a town in the State of Bahia, between January 2014 to August 2017. Data were collected between September 2017 and March 2018 at the caregivers' homes, using a sociodemographic form and the Katz, Zarit Burden Interview and Perceived Stress scales, which were analyzed using descriptive statistics and the Spearman's correlation coefficient. Results: The 44 family caregivers presented mild to moderate burden, which was significantly related to the caregiver's stress level and the degree of dependence of the stroke survivor. Conclusion: This data highlights the need for greater formal social support for stroke survivors and their family caregivers, through health actions that favor the independence of those with stroke sequelae and include the caregiver in the rehabilitation plan, especially in the emotional dimension, in order to reduce the caregiver's burden, thus improving the well-being of these individuals.

Key words: Stroke; Caregivers; Family; Nursing Care; Workload.

\section{RESUMEN}

Objetivo: Analizar la sobrecarga de los cuidadores familiares de los sobrevivientes de accidente cerebrovascular y factores relacionados. Material y Método: Estudio transversal y correlacional, realizado con muestra no probabilística, seleccionada por conveniencia, de cuidadores familiares de sobrevivientes de accidente cerebrovascular, atendidos en 17 Unidades Básicas de Salud de la zona urbana o atendidos en la única unidad hospitalaria pública de un municipio al interior del estado de Bahía (Brasil), entre enero de 2014 a agosto de 2017. Los datos se recolectaron entre septiembre de 2017 y marzo de 2018 en los domicilios de los cuidadores, utilizando un formulario sociodemográfico y las escalas Katz, Zarit Burden Interview y Perceived Stress, que se analizaron mediante estadística descriptiva y el coeficiente de correlación de Spearman. Resultados: Los 44 cuidadores familiares presentan sobrecarga leve a moderada, la cual se relacionó significativamente con el estrés del cuidador y el grado de dependencia del superviviente del accidente cerebrovascular. Conclusión: Este dato muestra la necesidad de un mayor apoyo social formal para los sobrevivientes de accidente cerebrovascular y sus cuidadores familiares, a través de acciones de salud que favorezcan la independencia de las personas por sus secuelas e incluyan al cuidador en el plan de rehabilitación, especialmente en el ámbito emocional, con el fin de reducir la carga sobre el cuidador, mejorando así el bienestar de estas personas.

Palabras clave: Accidente Cerebrovascular; Cuidadores; Familia; Atención de Enfermería; Carga de Trabajo.

Data de recepção: 21/09/2020

Data de aceitação: 16/04/2021

\section{INTRODUÇÃO}

O Acidente Vascular Cerebral (AVC) é uma das principais causas de morbimortalidade entre as doenças cardiovasculares no mundo. No Brasil, por exemplo, durante o período de 1990 a 2015 , o número de pessoas com AVC e a proporção de anos perdidos devido à incapacidade aumentaram expressivamente ${ }^{(1)}$. Isso significa que, após a alta hospitalar, uns maiores números de sobreviventes de
AVC retornarão para suas residências dependendo de cuidados, e consequentemente, haverá um maior número de cuidadores familiares responsáveis por sua reintegração na comunidade e por sua adaptaçáo nas atividades básicas. Sem o suporte ou apoio social adequado, a saúde do cuidador estará comprometida, o que favorecerá o surgimento da sobrecarga relacionada ao cuidado ${ }^{(2)}$.

Essa sobrecarga é a tradução do termo em inglês burden e representa um fardo resultante do cuidado 
contínuo. Dentre as principais manifestaçôes da sobrecarga no cuidador estáo a ansiedade, os sintomas depressivos (i.e., cansaço, tristeza, extrema sensibilidade, dificuldade para dormir e falta de motivação), os sintomas físicos (i.e., dores de cabeça e ostemusculares), o estresse (i.e., impacto nos gastos financeiros e atividade social reduzida) e a piora da qualidade de vida. Estes sentimentos ocasionam um grande sofrimento mental e estes cuidadores que não tem com quem compartilhálo. Estas alteraçóes também podem comprometer a qualidade do cuidado prestado as pessoas dependentes pós-AVC ${ }^{(3-6)}$.

Apesar dessas consequências, ainda não há no Brasil políticas públicas que garantam assistência aos cuidadores ou protocolos de capacitação para o cuidado. Além disso, poucos estudos brasileiros identificaram os fatores associados à sobrecarga do cuidador de sobreviventes ao $\mathrm{AVC}^{(7,}$ 8). Todavia, esses estudos foram realizados em outros contextos regionais, que, diferentemente de algumas localidades da região Nordeste, possuem melhores indicadores socioeconômicos e acesso aos recursos de reabilitação do AVC, que são fatores que interferem significativamente na sobrecarga do cuidador $^{(1)}$.

Pesquisas com cuidadores familiares na região Nordeste do Brasil são incipientes, e a sua realização é, portanto, relevante. Neste sentido, este estudo amplia o conhecimento acerca da problemática do cuidador em um contexto social diferente de outras localidades nacionais, permitindo comparar divergências e semelhanças inter-regionais entre o nível da sobrecarga e os seus fatores de riscos e proteção. Estes dados auxiliam na projeçáo de intervençóes em saúde, que podem ser programadas a partir das necessidades específicas desses cuidadores, possibilitando assim a melhora concomitante da saúde do cuidador e do cuidado adequado ao sobrevivente de $\mathrm{AVC}^{(9)}$.

Os enfermeiros podem estar à frente destas intervençôes, por meio do manejo de estratégias educativas resolutivas e inovadoras, assim como, na articulação intersetorial com outras redes de apoio social. Além disso, os dados desta pesquisa podem direcionar a criação de políticas públicas, que assegurem o direito de assistência integral a estes cuidadores no âmbito nacional e internacional, considerando que a sobrecarga é um fenômeno mundial.
A hipótese deste estudo é que há correlação entre a sobrecarga do cuidador e as variáveis do cuidador, do cuidado e do sobrevivente de AVC. Portanto, este estudo teve como objetivo analisar a sobrecarga dos cuidadores familiares dos sobreviventes de Acidente Vascular Cerebral e os fatores relacionados.

\section{MATERIAL E MÉTODO}

Trata-se de um estudo transversal e correlacional, realizado no município de Guanambi, interior do estado da Bahia, região Nordeste do Brasil, com médio Índice de Desenvolvimento Humano (IDH). Uma enfermeira especialista, que foi treinada pela primeira autora, realizou a coleta dos dados, através da aplicação de quatro instrumentos, entre setembro de 2017 a março de 2018.

O tamanho da amostra dos cuidadores familiares deste estudo foi definido pela técnica de amostragem não probabilística, do tipo conveniência, e a sua seleção ocorreu a partir da identificação dos sobreviventes de AVC com dependência funcional, que possuíam cadastro em uma das 17 Unidades Básicas de Saúde da zona urbana, ou fossem atendidos na única unidade hospitalar pública do município em estudo entre janeiro de 2014 à agosto de 2017. Para tanto, aplicou-se ao cuidador familiar a escala de Atividades Básicas de Vida Diária de Katz, que avalia a dependência ou independência em 6 funçóes (alimentação, controle de esfíncteres, transferência, capacidade de ir ao banheiro, vestirse e tomar banho). O escore varia de 0 a 6 pontos, no qual cada ponto equivale à resposta "sim", sendo a pessoa classificada como independente em todas as funçôes ou dependente de 1 a 6 funçôes ${ }^{(10)}$. Os sobreviventes de AVC independentes foram excluídos da pesquisa.

Após a identificação dos sobreviventes de AVC dependentes, selecionou-se os cuidadores familiares que atenderam aos seguintes critérios de inclusão: ser o cuidador familiar principal de uma pessoa dependente de cuidados após o AVC, ter idade maior ou igual a 18 anos, apresentar tempo de cuidado de 6 a 50 meses (fase crônica do AVC) e residir na zona urbana do município em estudo.

Para os cuidadores elegíveis que desejaram participar do estudo, aplicou-se um formulário semiestruturado para verificar as características sociodemográficas do binômio, saúde do cuidador 
e organização do cuidado. Posteriormente, verificou-se a sobrecarga do cuidador em relação aos cuidados prestados ao sobrevivente de AVC por meio da escala de Zarit Burden Interview, um instrumento composto por 22 perguntas, que avalia as áreas psicossociais e financeira. Cada pergunta desse instrumento é pontuada em uma escala tipo Likert de 5 pontos, cujas respostas variam de 0 (nunca) a 4 (sempre), a exceção é da última pergunta, na qual o cuidador responde o quanto se sente sobrecarregado por cuidar de seu familiar ( $0=$ nada a $4=$ extremamente). $\mathrm{O}$ escore $\mathrm{da}$ escala varia entre 0 e 88 . Assim, quanto maior a pontuação, maior a sobrecarga. Apesar de ter sido desenvolvida para cuidadores de pessoas idosas e com demência, essa escala pode ser aplicada em cuidadores de diversas doenças mentais e físicas. No Brasil, a sua consistência interna e validade foram testadas em cuidadores de idosos com depressão, cujo coeficiente alfa de Cronbach apresentou valor de $0,87^{(11)}$.

Posteriormente, aplicou-se a escala de Estresse Percebido, que foi testada e validada no Brasil em indivíduos de 18 a 70 anos $^{(12)}$. Esta escala é composta por 14 questóes do tipo Likert, com respostas que variam entre 0 e $4(0=$ nunca, $1=$ quase nunca, $2=$ às vezes, $3=$ pouco frequente e $4=$ muito frequente). A soma das questóes gera escores que variam de $0 \mathrm{a}$ 56 pontos, sendo que, quanto maior a pontuação, maior o estresse ${ }^{(13)}$.

O software Statistical Package for the Social Sciences (SPSS), versão 21.0, foi utilizada para todas as análises estatísticas, considerando o nível de significância de $5 \%(\mathrm{p}<0,05)$. A não normalidade dos dados foi verificada pelo teste de Shapiro-Wilk. Assim, os resultados das variáveis contínuas foram expressos em medianas e Intervalo Interquartil (IQ), e os das categóricas, em valores absolutos e porcentagens. No intuito de verificar a correlação entre a variável dependente (sobrecarga do cuidador) e as variáveis independentes vinculadas ao cuidador (idade, renda e estresse), cuidado (horas de cuidado) e sobrevivente de AVC (idade e grau de independência funcional pela escala de Katz), realizou-se a correlação de Spearman, classificada, de acordo com sua magnitude, em: <0,3 (fraca), $\geq$ $0,3 \mathrm{a}<0,5$ (moderada) e $\geq 0,5$ (forte) $)^{(14)}$.

O estudo foi aprovado pelo Comitê de Ética em Pesquisa da Universidade Estadual do Sudoeste da Bahia (UESB), campus de Jequiél Bahia, sob o protocolo 2.187.869 e CAAE 71341017.5.0000.0055. Todos os participantes formalizaram sua adesão por meio da assinatura do Termo de Consentimento Livre e Esclarecido (TCLE).

\section{RESULTADOS}

Dos 44 sobreviventes de AVC que recebiam cuidados, $54,5 \%$ eram homens, com mediana de idade de 76,50 anos (IQ=62,25-83,00). Os dados da escala de Katz mostram que a maioria dos sobreviventes de AVC (52,3\%) era dependente em 3 a 5 funçóes e que 22,7\% eram totalmente dependentes.

Quanto ao perfil sociodemográfico dos 44 cuidadores, verificou-se que a maioria era do sexo feminino (93,2\%), filhos/as e cônjuges $(84,1 \%)$, possuía companheiro/a $(68,2 \%)$, mediana de 49,50 anos de idade (variaçáo entre 18 e 77 anos), com até 9 anos de estudo (41,0\%), estava desempregada $(47,6 \%)$ e sobrevivia com menos de um salário mínimo (63,6\%). A maior parte dos cuidadores prestava cuidado por mais que 16 horas semanais, residia com o sobrevivente de AVC (90,9\%) e contava com a ajuda esporádica de outras pessoas $(56,8 \%)$ (Tabela 1$)$.

$\mathrm{O}$ escore da sobrecarga dos cuidadores variou de 5 a 71, enquanto o do estresse oscilou entre 3 e 45 pontos. O estresse do cuidador e o grau de dependência do sobrevivente de AVC foram as variáveis com correlação significativa forte e moderada com a sobrecarga do cuidador (Tabela 2). 
Tabela 1. Variáveis dos cuidadores familiares e dos sobreviventes de AVC na fase crônica da doença. Guanambi-BA, 2017-2018.

\begin{tabular}{|c|c|c|c|}
\hline Participantes do estudo & Variáveis & & Resultados \\
\hline \multirow{4}{*}{ Sobrevivente de AVC $(n=44)$} & \multicolumn{2}{|l|}{ Idade em anos ${ }^{a}$} & $76,50(62,25-83,00)$ \\
\hline & $\operatorname{Sexo}^{\mathrm{b}}$ & Feminino & $20(45,5)$ \\
\hline & & Masculino & $24(54,5)$ \\
\hline & Grau de independência ${ }^{a}$ & & $4,00(2,25-5,00)$ \\
\hline \multirow{28}{*}{ Cuidador familiar $(\mathrm{n}=44)$} & Idade em anos ${ }^{a}$ & & $49,50(37,25-63,00)$ \\
\hline & $\mathrm{Sexo}^{\mathrm{b}}$ & Feminino & $41(93,2)$ \\
\hline & & Masculino & $3(6,8)$ \\
\hline & Situação conjugal ${ }^{b}$ & $\begin{array}{l}\text { Com } \\
\text { companheiro(a) }\end{array}$ & $30(68,2)$ \\
\hline & & Sem companheiro(a) & $14(31,8)$ \\
\hline & $\operatorname{Renda}^{\mathrm{b}}$ (salário mínimo ${ }^{\mathrm{c}}$ ) & Menor que um & $28(63,6)$ \\
\hline & & Maior ou igual a um & $16(36,4)$ \\
\hline & Atividade laboral (Ocupação) ${ }^{\mathrm{b}}$ & Trabalha fora de casa & $9(20,5)$ \\
\hline & & Não trabalha & $21(47,6)$ \\
\hline & & Aposentado/a & $14(31,9)$ \\
\hline & Escolaridade (anos de estudo) ${ }^{\mathrm{b}}$ & Menor que 1 ano & $6(13,6)$ \\
\hline & & 1 a 9 anos & $18(41,0)$ \\
\hline & & 10 a 12 anos & $17(38,6)$ \\
\hline & & Maior que 12 anos & $3(6,8)$ \\
\hline & Grau de parentesco ${ }^{\mathrm{b}}$ & Filho/a & $20(45,5)$ \\
\hline & & Cônjuge & $17(38,6)$ \\
\hline & & Irmão/ã & $1(2,3)$ \\
\hline & & Pai/mãe & $2(4,5)$ \\
\hline & & Outro & $4(10,0)$ \\
\hline & $\begin{array}{l}\text { Reside com o sobrevivente de } \\
\text { AVC }^{b}\end{array}$ & $\operatorname{Sim}$ & $40(90,9)$ \\
\hline & & Não & $4(9,1)$ \\
\hline & Horas de cuidado por semana ${ }^{b}$ & Até 8 & $12(27,3)$ \\
\hline & & De 9 a 15 & $15(34,1)$ \\
\hline & & $\begin{array}{l}\text { Mais que } 16 \text { ou } \\
\text { tempo integral }\end{array}$ & $17(38,6)$ \\
\hline & Auxílio para o cuidado ${ }^{\mathrm{b}}$ & Sim & $25(56,8)$ \\
\hline & & Não & $19(43,2)$ \\
\hline & Sobrecarga do cuidador ${ }^{\mathrm{a}}$ & & $32,50(19,25-40,00)$ \\
\hline & Estresse do cuidador ${ }^{a}$ & & $24,50(18,00-32,75)$ \\
\hline
\end{tabular}

a= mediana, Intervalo Interquartil; b= número, porcentagem; c=salário mínimo ( $\mathrm{R} \$ 937,00$ reais) 
Tabela 2. Coeficiente de correlação de Spearman (r) entre a sobrecarga e as variáveis do cuidador, do cuidado e do sobrevivente de AVC, Guanambi-BA, 2019.

\begin{tabular}{|c|c|c|c|c|c|c|c|c|c|c|c|c|}
\hline \multicolumn{13}{|c|}{ Variáveis } \\
\hline & \multicolumn{2}{|c|}{ Katz } & \multicolumn{2}{|c|}{$\begin{array}{c}\text { PSSS } \\
\text { Estresse do } \\
\text { cuidador }\end{array}$} & \multicolumn{2}{|c|}{$\begin{array}{l}\text { Renda } \\
\text { Cuidador } \\
\text { Familiar }\end{array}$} & \multicolumn{2}{|c|}{$\begin{array}{l}\text { Horas de } \\
\text { cuidado }\end{array}$} & \multicolumn{2}{|c|}{$\begin{array}{c}\text { Idade } \\
\text { Cuidador } \\
\text { Familiar }\end{array}$} & \multicolumn{2}{|c|}{$\begin{array}{c}\text { Idade } \\
\text { sobrevivente } \\
\text { AVC } \\
\end{array}$} \\
\hline & $\mathrm{r}$ & $\mathrm{p}$ & $\mathrm{r}$ & $\mathrm{p}$ & $\mathrm{r}$ & $\mathrm{p}$ & $\mathrm{r}$ & $\mathrm{p}$ & $\mathrm{R}$ & $\mathrm{p}$ & $\mathrm{r}$ & $\mathrm{p}$ \\
\hline Zarit & $-0,358$ & $0,017^{*}$ & 0,502 & $0,001^{*}$ & 0,161 & 0,296 & $-0,124$ & 0,424 & 0,06 & 0,699 & $-0,052$ & 0,74 \\
\hline
\end{tabular}

${ }^{*}$ Correlação estatisticamente significativa $(\mathrm{p}<0,05)$

\section{DISCUSSÁO}

Este estudo demostrou que, mesmo após vários meses de retorno ao domicílio, os cuidadores familiares dos sobreviventes de AVC continuam sobrecarregados e que os aspectos emocionais podem influenciar significativamente no aumento dessa carga, pois o estresse é um elemento que permeia a inter-relaçáo do binômio durante o ato de cuidar. Do mesmo modo, apesar da moderada intensidade, o grau de dependência do sobrevivente de AVC constituiu um fator preditivo da sobrecarga do cuidador. A incapacidade funcional do paciente dificulta o cuidado cotidiano, pois os cuidadores precisam aprender a realizar novas atividades (por exemplo, transferência, administração de medicamentos, encontrar redes de apoio, entre outras atividades) ao mesmo tempo que cuidam das atividades domésticas, sem um adequado suporte social profissional, governamental e familiar, respectivamente.

Em consonância com estudos prévios nacional ${ }^{(8)}$ e internacionais ${ }^{(15,16)}$, os cuidadores, em sua maioria, são mulheres, casadas, adultas de meia-idade ou idosas, filhas ou cônjuges dos sobreviventes de AVC. Isso mostra que a cultura do cuidado feminino prevalece na sociedade contemporânea, ancorada nos valores morais construídos historicamente. A mulher enquanto mãe é naturalmente uma cuidadora; portanto, cuidar de outros membros da família diante de uma situação de adoecimento é percebido como uma extensão altruísta de seu papel e náo como um trabalho.

Embora haja uma participação do sexo masculino no cuidado de seus familiares enfermos, conforme dados deste $(6,8 \%)$ e de outros estudos
$(10 \text { e } 16,3 \%)^{(8,17)}$, esse auxílio ainda é modesto. Isso decorre, principalmente, da cultura machista e de a função da manutenção financeira do lar ser predominantemente masculina.

Em outras culturas, como na África do Sul ${ }^{(18)}$, foi identificado que o cuidado era realizado, em sua maioria, pelos homens, devido ao sistema familiar unificado após o matrimônio, no qual o homem promove a maior parte da assistência. Contudo, nesses exemplos, ser do sexo masculino não foi associado a sobrecarga do cuidado. Isso pode ter ocorrido porque os homens desses referidos estudos foram recrutados em unidades hospitalares, onde o tempo de cuidado é menor e há auxílio profissional. Contrariamente a este contexto, na residência, as mulheres cuidam dos sobreviventes de AVC por um período maior e têm escasso apoio social.

A maior idade do cuidador familiar do sobrevivente de AVC já foi constatada em estudos anteriores, principalmente entre os cônjuges e filhos $^{(8,17,19,20)}$. No presente estudo, a mediana de idade foi de 49,50 anos, com a maioria dos cuidadores sendo filhas/os (45,5\%) casadas/os e esposas/os (38,6\%), o que pode explicar a variação da idade de 18 a 77 anos. Esses cuidadores geralmente assumem um papel mais proativo nessa função em relação aos cuidadores solteiros e mais jovens ${ }^{(20)}$, pois estáo interligados pelo voto matrimonial ou pela responsabilidade filial. Essa atitude potencializa o risco de sobrecarga em uma idade avançada, na qual a saúde já está fragilizada pelo processo de envelhecimento e pelo aparecimento de doenças crônicas em adultos, sobretudo as hereditárias, as genéticas e as relacionadas ao estilo de vida não saudável.

Assim como em outros estudos ${ }^{(21,22)}$, os cui- 
dadores pesquisados apresentaram baixo nível educacional, sendo que a maioria $(54,6 \%)$ possuía até 9 anos de estudo. Mesmo em países desenvolvidos, com melhores sistemas de educação, como o Canadá(15) e os Estados Unidos da América ${ }^{(23)}$, os cuidadores que possuem de médio a alto nível de escolaridade encontram-se com intensidade de sobrecarga de moderada a alta, respectivamente. É provável que os cuidadores com menor grau de escolaridade apresentam dificuldades em compreender as orientaçóes dos profissionais de saúde ${ }^{(17,19,24)}$. De maneira oposta, os cuidadores com maior grau de instrução podem ter maior prejuízo no âmbito emocional da sobrecarga, devido à compreensão de sua limitada possibilidade de intervenção perante a gravidade do AVC, que pode ter sequelas irreversíveis, apesar do maior acesso às tecnologias em saúde de média e alta complexidade.

No que se refere a atividade laboral, os cuidadores que trabalhavam fora de casa era uma minoria $(20,5 \%)$ e enfrentavam uma dupla jornada de trabalho ao retornar ao domicílio, pois dedicavam maior tempo de cuidado com atividades mais complexas ao sobrevivente de AVC em relação aos demais familiares. Este excesso de atividades dentro e fora do domicílio possui efeitos negativos na saúde física e emocional dos cuidadores, o que contribui para o aumento da sobrecarga de cuidado ${ }^{(3)}$.

Todavia, estudos também mostram que o desemprego e escassos recursos financeiros, oriundos da dedicação exclusiva ao sobrevivente de AVC, prejudicam o cuidador. Para aqueles que são adultos jovens e de meia-idade, essa dedicação compromete o seu retorno ao mercado de trabalho e a sua aposentadoria ${ }^{(25)}$. Semelhante a estudos $\operatorname{prévios}^{(8,}$ 17), a maioria dos cuidadores estava desempregada e dependendo financeiramente de outros membros da família. Este é um cenário comum entre os cuidadores e o suporte de um assistente social seria fundamental para orientar sobre a busca de recursos sociais na rede assistencial, como o auxílio financeiro, empréstimo ou doação de produtos (i.e., cadeira de rodas, camas, alimentação), entre outros. Este suporte social informativo pode contribuir para reduzir a sobrecarga e melhorar a qualidade de vida dos cuidadores familiares ${ }^{(26)}$.

Essa precária situação laboral ainda compromete sua renda financeira, pois a maior parte dos cuidadores estudados sobreviviam com menos de um salário mínimo $(63,6 \%)$, de modo semelhante a outras pesquisas ${ }^{(8,17)}$. Diante da necessidade de cuidados à própria saúde, esses cuidadores precisam enfrentar longas filas de espera para o atendimento nos serviços públicos ${ }^{(8)}$. Por outro lado, os cuidadores com maior renda financeira podem se manter em seus empregos e pagar por melhores recursos de saúde, o que possibilita que se adaptem aos desafios do cuidado, contribuindo para uma menor sobrecarga objetiva ${ }^{(23)}$.

Elevado percentual dos cuidadores residem com os sobreviventes de AVC (90,9\%), o que corrobora com estudos prévios $^{(9,19)}$. Coabitar com a pessoa dependente pode facilitar as atividades cuidativas, mas, por outro lado, pode ser um fator gerador de estresse. Não obstante, o desgaste mental e físico do cuidador pode resultar em violência ao sobrevivente de AVC, especialmente contra os idosos e aqueles com maior déficit cognitivo e funcional, o que revela a necessidade de distribuir o cuidado entre os familiares.

A maioria dos cuidadores relatam que recebem ajuda de seus familiares $(56,8 \%)$, tal como em estudos anteriores, cujos principais suportes recebidos foram o financeiro, nas tarefas cotidianas relacionadas ao paciente, como alimentação e acompanhamento nas consultas e exames nas unidades de saúde, assim como em atividades domésticas relacionadas à limpeza da casa ou cuidar dos filhos ${ }^{(6,20)}$. Contudo, como geralmente esse suporte é pontual, os cuidadores mantêm-se sobrecarregados $^{(9,27)}$.

A sobrecarga da amostra estudada, mensurada pela escala de Zarit, foi maior que em outros estudos (internacional e nacional), também realizado na fase crônica do $\mathrm{AVC}^{(3,8)}$. Tal fato pode ser atribuído ao grau de dependência dos sobreviventes de AVC e às horas de cuidado serem maiores, assim como as diferentes condiçóes socioeconômicas das regióes onde os estudos foram desenvolvidos.

Quanto aos sintomas psicopatológicos, o estresse é uma alteração comum aos cuidadores de pacientes com doenças crônicas incapacitantes, como o $\mathrm{AVC}^{(28)}$. Neste estudo, entre as variáveis analisadas, o estresse apresentou valor superior a outra pesquisa com cuidadores informais ${ }^{(29)}$ e correlação positiva forte com a sobrecarga desses cuidadores, semelhantemente a outros estudos internacionais ${ }^{(18,}$ 30) e a uma pesquisa nacional ${ }^{(31)}$, destacando que o estado emocional possui influência importante no 
aumento da carga de cuidado, mesmo em diferentes contextos geográficos.

A correlação entre essas variáveis (sobrecarga e estresse) pode decorrer de estratégias ineficientes de enfrentamento dos problemas cotidianos provenientes de conflitos internos (i.e., culpa por sentimentos negativos como violência, raiva e alteraçôes do humor) e interpessoais (cuidadorpaciente-família-comunidade) ou na suspensão de atividades pessoais e prazerosas. Dessa maneira, os profissionais de saúde devem atentar para intervir ${ }^{(32)}$ na prevenção ou na redução desse fardo, uma vez que estudo anterior mostrou uma chance de sobrecarga 2,27 vezes maior em cuidadores com apoio social emocional insatisfatório em comparação com o satisfatório $^{(9)}$. A correlação forte encontrada entre o estresse e a sobrecarga do cuidador indica que a saúde mental deve ser um das prioridades dos profissionais de saúde no cuidado ao cuidador.

$\mathrm{Na}$ amostra estudada, foi encontrada ainda uma correlação moderada significativa entre o grau de dependência do sobrevivente de AVC e a sobrecarga do cuidador, tal como em estudo prévio $^{(20)}$, principalmente quando estes cuidam de familiares com maior comprometimento das funçôes motoras. Esta correlação indica que o cuidado à saúde do cuidador também perpassa pela necessidade de assistência precoce e contínua ao sobrevivente de AVC, sobretudo nas unidades de saúde e setores vinculados.

Esta pesquisa apresenta algumas limitações por se tratar de um estudo transversal, com amostra de uma determinada localidade. Estudos longitudinais com maior amostra e em diferentes regiôes são necessários para avaliar a correlação entre essas variáveis em diferentes áreas geográficas e momentos no tempo.

Faz-se necessário ofertar mais apoio formal aos sobreviventes de AVC para reduzir a sua dependência e incorporar os cuidadores ao plano de reabilitação de seus familiares, oferecendo-lhes também uma atenção adequada e intervençôes precoces, que enfoquem ações educativas para a resolução dos problemas e do controle de emoçóes, desde o diagnóstico da doença até a fase atual do cuidado, com o intuito de reverter os fatores relacionados à sobrecarga. Essas açôes destinadas à díade podem ser gerenciadas por enfermeiros em parceria com uma equipe multiprofissional, priorizando o atendimento de reabilitação do sobrevivente de
AVC e criando-se grupos de apoio aos cuidadores nas unidades de atenção primária à saúde ou podem acontecer enquanto estes aguardam o término das sessóes de reabilitação dos seus familiares.

\section{CONCLUSÓES}

Este estudo evidencia que a maioria dos cuidadores é composta por pessoas casadas, do sexo feminino, cônjuges e filhas, tem baixa escolaridade e renda, não possui ocupação e reside com o sobrevivente de AVC, o que representa características semelhantes à de outros cuidadores familiares nos cenários nacional e internacional.

Os fatores relacionados com a sobrecarga destes cuidadores foram o próprio estresse e o declínio funcional dos pacientes, que apresentam escores superiores aos de outros estudos com cuidadores informais. Os enfermeiros podem proporcionar apoio social formal, por meio de aconselhamento psicológico e orientaçóes para o cuidado domiciliar pós-AVC para minimizar o sofrimento mental destes cuidadores e aumentar o grau de independência dos sobreviventes de AVC. Estas medidas podem auxiliar na redução ou prevenção da sobrecarga de cuidado, uma vez que os dados revelam a necessidade de açôes conjuntas em saúde que priorizem a assistência à díade cuidador-pessoa cuidada durante o cuidado continuum.

\section{AGRADECIMENTO}

Aos cuidadores familiares dos sobreviventes de AVC que participaram deste estudo.

\section{REFERÊNCIAS}

1. Lotufo PA, Goulart AC, Passos VMA, Satake FM, Souza MFM, França EB, et al. Doença cerebrovascular no Brasil de 1990 a 2015: Global Burden of Disease 2015. Rev Bras Epidemiol [Internet]. 2017 [citado 2020 mai 22]; 20 (supl. 1): 129-41. Disponível em: https://www.scielo. $\mathrm{br} / \mathrm{j} / \mathrm{rbepid} / \mathrm{a} / 5 \mathrm{~K} 9 \mathrm{xPnDV}$ szVTG9CYQT7wzgD/?1 ang $=$ pt $\&$ format $=$ pdf

2. Pallesen H, Næss-Schmidt ET, Kjeldsen SS, Pedersen SKS, Sørensen SL, Brunner I, et al. Stroke - 65 Plus. Continued Active Life: a study protocol 
for a randomized controlled cross-sectoral trial of the effect of a novel self-management intervention to support elderly people after stroke. Trials [Internet]. 2018 [citado 2019 nov 19]; 19(1): 639. Disponível em: https://pubmed.ncbi.nlm.nih. gov/30454014/

3. Hu P, Yang Q, Kong L, Hu L, Zeng L. Relationship between the anxiety/depression and care burden of the major caregiver of stroke patients. Medicine [Internet]. 2018 Oct [citado 2021 abr 4]; 97(40): e12638. Disponível em: https://www.ncbi.nlm. nih.gov/pmc/articles/PMC6200450/

4. Loh AZ, Tan JS, Zhang MW, Ho RC. The global prevalence of anxiety and depressive symptoms among caregivers of stroke survivors. J Am Med Dir Assoc [Internet]. 2017 [citado 2021 abr 4]; 18(2): 111-6. Disponível em: https://www.jamda. com/article/S1525-8610(16)30366-8/pdf

5. Gomes MLP, Silva JCB, Batista EC. Escutando quem cuida: quando o cuidado afeta a saúde do cuidador em saúde mental. Rev Psicol Saúde [Internet]. 2018 [citado 2021 abr 4]; 10(1): 3-7. Disponível em: http://pepsic.bvsalud.org/ scielo.php?script $=$ sci_arttext $\&$ pid $=$ S2177-093 $\mathrm{X} 2018000100001 \& \operatorname{lng}=\mathrm{pt}$

6. Pesantes MA, Brandt LR, Ipince A, Miranda JJ, Diez-Canseco F. An exploration into caring for a stroke-survivor in Lima, Peru: Emotional impact, stress factors, coping mechanisms and unmet needs of informal caregivers. eNeurologicalSci [Internet]. 2017 [citado 2018 jun 9]; 6: 33-50. Disponível em: https://www.sciencedirect.com/science/article/ pii/S2405650216300363

7. Costa TF, Costa KNFM, Martins KP, Fernandes MGM, Brito SS. Sobrecarga de cuidadores familiares de idosos com acidente vascular encefálico. Esc Anna Nery [Internet]. 2015 [citado 2018 abr 6]; 19(2): 350-355. Disponível em: https://www.scielo.br/scielo.php?script=sci_ arttext\&pid $=$ S1414-81452015000200350

8. Caro CC, Costa JD, Da Cruz DMC. Burden and Quality of Life of Family Caregivers of Stroke Patients. Occup Ther Health Care [Internet]. 2018 [citado 2018 apr 6]; 32(2): 154-71. Disponível em: https://pubmed.ncbi.nlm.nih.gov/29578827/

9. Lino VTS, Rodrigues NCP, Camacho LAB, O’Dwyer G, Lima IS, Andrade MKN, et al. Prevalência de sobrecarga e respectivos fatores associados em cuidadores de idosos dependentes, em uma regiáo pobre do Rio de Janeiro, Brasil. Cad Saúde Pública [Internet]. 2016 [citado 2019 fev 1]; 32(6): e00060115. Disponível em: https:// doi.org/10.1590/0102-311X00060115

10. Lino VTS, Pereira SRM, Camacho LAB, Ribeiro Filho ST, Buksman S. Adaptação transcultural da Escala de Independência em Atividades da Vida Diária (Escala de Katz). Cad Saúde Pública [Internet]. 2008 [citado 2020 ago 30]; 24(1):10312. Disponível em: https://www.scielo.br/j/csp/a/ hssCqfGkZRfBCH5Nc9fBbtN/?lang=pt \& format=pdf

11. Scazufca M. Versão brasileira da escala Burden Interview para avaliação de sobrecarga em cuidadores de indivíduos com doenças mentais. Rev Bras Psiquiatr [Internet]. 2002 [citado 2018 abr 06]; 24(1):12-7. Disponível em: https://www.scielo.br/j/ rbp/a/W8TVd9skgdCrM7vCJDjJrRh/?lang=en

12. Faro, A. Análise fatorial confirmatória das três versões da Perceived Stress Scale (PSS): um estudo populacional. Psicol Reflex Crit [Internet]. 2015 [citado 2018 abr 13]; 28(1): 21-30. Disponível em: https://doi.org/10.1590/1678-7153.201528103

13. Luft CDB, Sanches SO, Mazo GZ, Andrade A. Versão brasileira da Escala de Estresse Percebido: tradução e validação para idosos. Rev Saúde Pública [Internet]. 2007 [citado 2018 mai 9]; 41(4): 60615. Disponível em: https://www.scielo.br/scielo. php?script=sci_arttext\&pid=S0034-89102007000 400015

14. Oliveira DC, Carvalho GSF, Stella F, Higa CMH, D'Elboux MJ. Qualidade de vida e sobrecarga de trabalho em cuidadores ambulatoriais de idosos. Texto \& Contexto Enferm [Internet]. 2011 [citado 2020 set 23]; 20(2): 234-40. Disponível em: https:// doi.org/10.1590/S0104-07072011000200003

15. Mores G, Whiteman RM, Ploeg J, Knobl P, Cahn M, Klaponski L, et al. An Evaluation of the Family Informal Caregiver Stroke Self-Management Program. Can J Neurol Sci [Internet]. 2018 [citado 2020 apr 3]; 45(6): 660-8. Disponível em: https:// pubmed.ncbi.nlm.nih.gov/30430966/

16. Christensen ER, Golden SL, Gesell SB. Perceived Benefits of Peer Support Groups for Stroke Survivors and Caregivers in Rural North Carolina. N C Med J [Internet]. 2019 [citado 2020 oct 7]; 80(3): 143-8. Disponível em: https://www. ncmedicaljournal.com/content/80/3/143

17. Ugur HG, Erci B. The Effect of Home Care for Stroke Patients and Education of Caregivers on the Caregiver Burden and Quality of Life. Acta Clin Croat [Internet]. 2019 [citado 2021 apr 6]; 58(2): 321-32. Disponível em: https://www.ncbi.nlm. nih.gov/pmc/articles/PMC6884374/

18. Gbiri CA, Olawale OA, Isaac SO. Stroke management: informal caregivers'burdens and strains of caring for stroke survivors. Ann Phys Rehabil Med [Internet]. 2015 [citado 2018 mar 7]; 58(2): 98-103. Disponível em: https://pubmed. ncbi.nlm.nih.gov/25752228/

19. Menon B, Salini P, Habeeba K, Conjeevaram J, 
Munisusmitha K. Female Caregivers and Stroke Severity Determines Caregiver Stress in Stroke Patients. Ann Indian Acd Neurol [Internet]. 2017 [citado 2019 mar 27]; 20(4): 418-24. Disponível em: https://pubmed.ncbi.nlm.nih.gov/29184350/

20. Hekmatpou D, Baghban EM, Dehkordi LM. The effect of patient care education on burden of care and the quality of life of caregivers of stroke patients. J Multidiscip Healthc [Internet]. 2019 [citado 2020 oct 18]; 12: 211-17. Disponível em: https://pubmed.ncbi.nlm.nih.gov/30936715/

21. Kamwesiga JT, Eriksson GM, Tham K, Fors U, Ndiwalana A, von Koch L, et al. A feasibility study of a mobile phone supported family-centred ADL intervention, $\mathrm{F} @ \mathrm{ce}^{\mathrm{TM}}$, after stroke in Uganda. Global Health [Internet]. 2018 [citado 2020 nov 13]; 14:82. Disponível em: https://www.ncbi.nlm. nih.gov/pmc/articles/PMC6094578/

22. Parra MD, Torres CC, Arboleda LB, Carvajal RR, Franco S, Santos J. Effectiveness of an Educational Nursing Intervention on Caring Ability and Burden in Family Caregivers of Patients with Chronic Non-Communicable Diseases. A Preventive Randomized Controlled Clinical Trial. Invest Educ Enferm [Internet]. 2019 [citado 2020 set 21]; 37(1): e04. Disponível em: https://pubmed.ncbi. nlm.nih.gov/31083841/

23. Graf R, LeLaurin J, Schmitzberger M, Freytes IM, Orozco T, Dang $S$, et al. The stroke caregiving trajectory in relation to caregiver depressive symptoms, burden, and intervention outcomes. Top Stroke Rehabil [Internet]. 2017 [citado 2018 dec 19]; 24(7): 488-95. Disponível em: https:// pubmed.ncbi.nlm.nih.gov/28618848/

24. Jesus ITM, Orlandi AAS, Zazzetta MS. Sobrecarga, perfil e cuidado: cuidadores de idosos em vulnerabilidade social. Rev Bras Geriatr Gerontol [Internet]. 2018 [citado 2020 nov 1]; 21(2):194-204. Disponível em: https://doi. org/10.1590/1981-22562018021.170155

25. Schulz CH, Godwin KM, Herschc GI, Hyde LK, Irabor JJ, Ostwald SK. Return to work predictors of stroke survivors and their spousal caregivers. Work [Internet]. 2017 [citado 2020 mar 14]; 57(1): 11124. Disponível em: https://pubmed.ncbi.nlm.nih. gov/28506018/

26. Lehnerer S, Hotter B, Padberg I, Knispel P, Remstedt D, Liebenau A, et al. Social work support and unmet social needs in life after stroke: a cross-sectional exploratory study. BMC Neurol [Internet]. 2019 [citado apr 6]; 19: 220. Disponível em: https://pubmed.ncbi.nlm.nih.gov/31492151/

27. Delalibera M, Presa J, Barbosa A, Leal I. Sobrecarga no cuidar e suas repercussóes nos cuidadores de pacientes em fim de vida: revisão sistemática da literatura. Cienc Saúde Colet [Internet]. 2015 [citado 2018 dec 7]; 20(9): 2731-47. Disponível em: https://doi.org/10.1590/141381232015209.09562014

28. Cesário VAC, Leal MCC, Marques APO, Claudino KA. Estresse e qualidade de vida do cuidador familiar de idoso portador da doença de Alzheimer. Saúde debate [Internet]. 2017 [citado 2019 jan 2]; 41(112): 171-82. Disponível em: https://doi. org/10.1590/0103-1104201711214

29. Oliveira NA, Souza EN, Luchesi BM, Inouye K, Pavarini SCI. Estresse e otimismo de idosos que cuidam de idosos e vivem com crianças. Rev Bras Enferm [Internet]. 2017 [citado 2019 nov 13]; 70(4): 697-703. Disponível em: https://doi. org/10.1590/0034-7167-2017-0088

30. Lai S-T, Tan W-Y, Wo MC-M, Lim K-S, Ahmad $\mathrm{SB}$, Tan C-T. Burden in caregivers of adults with epilepsy in Asian families. Seizure [Internet]. 2019 [citado 2020 apr 28]; 71: 132-9. Disponível em: https://pubmed.ncbi.nlm.nih.gov/31325820/

31. Souza LR, Hanus JS, Libera LBD, Silva VM, Mangilli EM, Simões PW, et al. Sobrecarga no cuidado, estresse e impacto na qualidade de vida de cuidadores domiciliares assistidos na atenção básica. Cad Saúde Colet [Internet]. 2015 [citado 2018 set 17]; 23(2): 140-9. Disponível em: https:// doi.org/10.1590/1414-462X201500020063

32. Viegas LM, Fernandes AMA, Veiga MAPL. Intervenção de enfermagem para gestão de estresse em família cuidadores de idosos dependentes adultos: um estudo piloto. Rev Baiana Enferm [Internet]. 2018 [citado 2020 set 14]; 32: e25244. Disponível em: https://periodicos.ufba.br/index. php/enfermagem/article/view/25244 